\title{
Building Very Large Complex Shapes using a Flexible Blade Cutter
}

\author{
Imre Horváth (i.horvath@io.tudelft.nl) \\ Delft University of Technology, The Netherlands \\ Joris S. M. Vergeest, Zoltán Rusák, Zsolt Kovács and György Kuczogi \\ Delft University of Technology, The Netherlands
}

Key words: Free-form cutting, thick layered fabrication, polynomial shape approximation, hot blade tool, tool path calculation

Abstract: In order to fabricate very large objects of unlimited complexity from various high density polymers, a thick layered LOM approach has been developed. The layers are individually cut by a heated flexible blade manipulated by a sculpting robot and then stacked together. The shape of the blade is continuously adapted to the target geometry for the layer that allows extremely thick layers. The TLOM technology is a multilevel optimization problem, involving 1) finding the shape of the blade that suffices to the minimum energy principle, 2) minimizing the deviation between shape curves on the layer and the blade's profile curve, 3) finding the best fitting region of the profile curves to various curved domains, 4) finding the best tool positions and the optimum tool path. We have developed numerical methods that enable us to investigate feasibility of thick-layered fabrication of various products. Graphical simulation of the machining process demonstrates the practical applicability of the TLOM technology to automatic shape fabrication.

\section{BRIEF INTRODUCTION TO LAYERED OBJECT MANUFACTURING}

Physical concept modeling has proved to be an effective solution to improve the outcome of conceptual design and to reduce the time of product development. In the field of industrial design, the requested physical models are large sized, structurally and morphologically complex, and they are expected to support activities other than shape presentation only. For instance, when produced as functioning prototypes, physical models can also be used for preliminary checking of the requested functions, manufacturability, assemblability, aesthetic and ergonomic properties. Physical concept models are generally made from plastic foam, paper, plywood, etc. by an incremental technology that is called layered object manufacturing (LOM). When the size of the physical models are above $0.6 \mathrm{~m} \times 0.6 \mathrm{~m} \times 0.6 \mathrm{~m}$ most of the conventional LOM technologies fail due to their size, performance and quality limitations. It regards to laser stereolitography, 3D-printing, selective laser sintering, fused filament deposition, etc. Therefore, the objective of our research and development has been to come up with an effective technology for fabrication of large sized, free-form physical models of various soft materials based on higher order shape approximation.

There are four typical phases of fabrication of very large sized, plastic foam objects. These are (a) shape decomposition, (b) layer thickness calculation, (c) layer manufacturing, and (d) object recomposition. Shape decomposition involves (a) separation of the object into individual components, (b) segmentation of the components according to their morphological properties, (c) definition of layers based on shape approximation, and (d) sectoring the layers due to manufacturability and/or morphological reasons, when applicable. The approximation assumes a set of layers of predetermined thickness and applies them in an adaptive way to minimize the deviation between the nominal shape and the approximating shape. Layer thickness calculation is an optimization process involving activities such as (a) determination

The original version of this chapter was revised: The copyright line was incorrect. This has been corrected. The Erratum to this chapter is available at DOI: 10.1007/978-0-387-35392-0_40 
of the optimal slicing position, (b) detaching the unfavorable slicing domains, (c) layer thickness allocation with a view to preciseness and process characteristics, and (d) placing inserts and auxiliary parts. Layer manufacturing comprises (a) blank cutting, and (b) front surface cutting. The recomposition subprocess consists of (a) finding optimal assembly position, (b) layer positioning and fixing, and (c) finishing and decoration of the physical model/prototype.

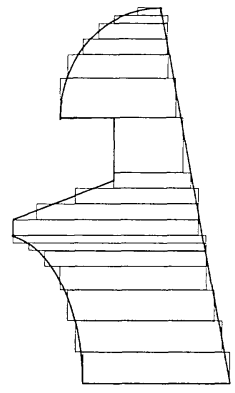

a. zero-order

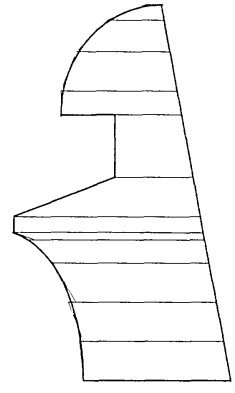

b. first-order

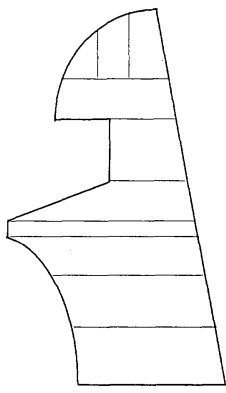

c. higher-order

Figure 1. Comparison of adaptive slicing techniques of various order

Various types of approximation methods have already been worked out. They can be classified based on the curvedness of the approximating curve/surface (Figure 1). The stepped approximation which is often referred to as zero-order approximation in literature is the most adequate for thin-layered deposition technologies. When the concept of uniform slicing is applied, it results in large errors of approximation for double-curved objects. In order to reduce the deviations, the concept of adaptive slicing has been introduced ${ }^{19,15}$. A stepwise uniform refinement of adaptive slicing was also presented ${ }^{18}$. Kulkarni, P. and Dutta, D. addressed the issue of containment to improve slicing ${ }^{16}$.

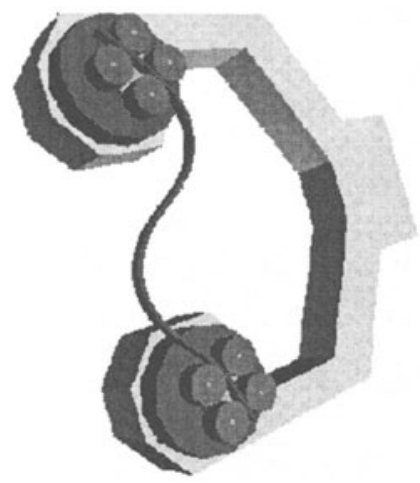

Figure 2. The flexible blade tool

To lessen the stair-case effect that is typical for stepped approximation, the concept of ruled/sloped approximation has been introduced ${ }^{3}$. This technique is also known as first order approximation in literature. Object fabrication with layers of ruled front surface was extensively studied ${ }^{6,7}$. De Jager P. J. et al. made a comparison between zero order and first order approximation techniques ${ }^{2}$. Slicing calculations are typically based on .STL files that however introduce errors in approximation of the nominal shape. To avoid representation 
errors techniques for direct slicing of CAD models have been worked out ${ }^{21,11}$. Guduri, S. et al. also addressed the issue of generating exact contour files ${ }^{5}$. First order shape approximation is generally combined with adaptive slicing. Segmentation is typically applied to the geometric model when the shape has morphologically dissimilar domains that need significantly different layer thickness. Special slicing techniques, e.g., sloped, and pie-like, can also be applied to achieve the same goal.

Involved in the Integrated Concept Advancement (ICA) research program, the authors have developed a new technology which is apparently the most appropriate for free-form, thick-layered fabrication of objects from high density plastic foams. The tool used for free form manufacturing of the front surfaces of the layers has been called flexible blade tool. The assembly model of the tool can be seen in Figure 2. The cross section of the blade is either circular or diamond shaped. The blade of pre-computed length is supported by pairs of electromechanically controlled rollers that allow us to set and instantaneously modify the clamping positions and, thus, to change the shape of the blade continuously.

In the further part of the paper the layer manufacturing process will be referred to as hot blade cutting. The whole process of shape decomposition, layer thickness calculation, layer cutting and stacking is called thick layered object manufacturing (TLOM). When it is applied to sculpted object we are talking about FF-TLOM where FF stands for free-form. Its concept, principles and the methodology of finding the shape of the flexible blade have been reported in an earlier paper with the contribution of some of the present authors. Due to the difficulties of a physically-based computation a geometrically-based modeling was applied ${ }^{10}$. Certain aspects of tool profile calculation, tool position and tool path calculation have also been presented in a former study ${ }^{9}$. This paper reports on the algorithms for computing and visualization of (a) the shape of the profile curve matching to a given domain of the front surface of the layer, (b) slicing the object into thick layers, (c) reduction of the cusp height when the calculated layer thickness is substituted by standard one, (d) determination of the tool positions to be set and calculation of the tool path, (e) virtual execution and verification of layer fabrication by process simulation. The free-form layer manufacturing process has been implemented on a six-axis sculpturing robot that is in use at the Section of Applied Information Technology, Sub-faculty of Industrial Design Engineering, of the Delft University of Technology.

\section{ALGORITHM FOR CALCULATION OF PROFILE CURVES OF THE FLEXIBLE BLADE}

Compared to the conventional layer cutting techniques of zero or first order, the hot blade cutting process is featured by a blade of instantaneously changing curvature. Consequently, the very first problem is to find the shape of the blade that provides the best fitting to the actual shape of the $\mathrm{CAD}$ geometry in the domain that corresponds to the layer thickness. The bent physical blade can be substituted by a curve of minimal elastic energy for given support conditions and for prescribed length. For calculation of this minimal energy curve various analytical ${ }^{4}$, variational ${ }^{17}$, and spline ${ }^{1}$ approximation algorithms have been published. Most of the spline fitting algorithms minimize the square of the second derivative along the curve ${ }^{8,20}$. To obtain the requested tool profile, we applied a further developed version of Kallay's algorithm ${ }^{13}$ which starts out of a point set, rather than of splines, in order to keep the method simple (Figure 3). When the flexible blade is in its minimal energy state, its shape takes up the smoothest curve for given support conditions. Therefore, this is the primary geometric requirement for interpolation of an arbitrary set of points.

The profile curve approximation algorithm starts out of the following assumptions: Let two points $\mathbf{p}_{\mathrm{i}} \neq \mathbf{p}_{\mathrm{k}}$ and two unit vectors $\mathbf{t p}_{\mathrm{i}}$ and $\mathrm{tp}_{\mathrm{k}}$ be given in the plane. Without loosing generality we may assume that the curve $\mathbf{r}$ that we search for is a mapping $\mathbf{r}(u)$ from the unit 
interval $[0,1]$ to the plane. We are looking for a curve $\mathbf{r}(u)$ which meets the following conditions: $\mathbf{r}(0)=\mathbf{p}_{\mathrm{i}}$ and $\mathbf{r}(1)=\mathbf{p}_{\mathrm{k}}, \dot{\mathbf{r}}(0)=a \mathbf{t}_{\mathrm{p}_{\mathrm{i}}}$ and $\dot{\mathbf{r}}(1)=d \mathbf{t}_{\mathrm{p}_{\mathrm{k}}}, \int_{0}^{1}|\dot{\mathbf{r}}(u)| d u=L$ and $\int_{0}^{1}|\kappa(u)|^{2} d u$ is minimal for any positive scalars $a$ and $d$. The last expression minimizes the strain energy of $\mathbf{r}$, which is proportional to the integrated squared curvature. Our objective is to have a calculation procedure that is independent of the material and stiffness (EI) properties of the blade. We used a selected set of points for the approximation. The vectors drawn between two neighboring points of the initial set of points are gradually rearranged towards a minimal energy configuration by subsequent spatial rotations, unless the curve is quasi-planar when rotations are applied in plane.

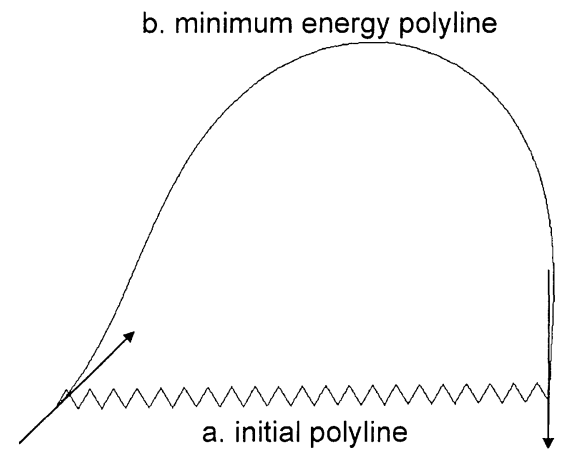

Figure 3. Start and end stage of curve stretching

Let's denote the vector between two points $\mathbf{p}_{\mathrm{i}}$ and $\mathbf{p}_{\mathrm{i}+1}$ by $\mathbf{u}_{\mathrm{i}+1}$. Since the lengths of the vectors have to be constant, $\sum_{i=1}^{n-1} \kappa\left(\mathbf{p}_{\mathrm{i}}\right)^{2}$ is minimal when the $U=\sum_{i=1}^{n} \mathbf{u}_{\mathrm{i}} \cdot \mathbf{u}_{\mathrm{i}+1}$ is maximal. To preserve the constant lengths of the vectors a special strategy of rotation is to be applied. Whenever we want to increase the value of $U$, all points $\mathbf{p}_{\mathrm{j}}$ between $\mathbf{p}_{\mathrm{i}}$ and $\mathbf{p}_{\mathrm{k}}$ has to be rotated about the axis $\mathbf{p}_{\mathrm{i}}-\mathbf{p}_{\mathrm{k}}$ by an angle $\alpha$ such that $\mathbf{u}_{\mathrm{i}} \mathbf{u}_{\mathrm{i}+1}+\mathbf{u}_{\mathrm{k}} \mathbf{u}_{\mathrm{k}+1}$ be maximal, under the condition that $\mathbf{u}_{\mathrm{i}-1}$ and $\mathbf{u}_{\mathrm{k}+1}$ remain unchanged. It means we have to minimize the complementary angles enclosed between the vectors $\mathbf{p}_{\mathrm{i}}-\mathbf{p}_{\mathrm{i}-1}$ and $\mathbf{p}_{\mathrm{i}+1}-\mathbf{p}_{\mathrm{i}}$, and $\mathbf{p}_{\mathrm{k}}-\mathbf{p}_{\mathrm{k}-1}$ and $\mathbf{p}_{\mathrm{k}+1}-\mathbf{p}_{\mathrm{k}}$. This method will increase $U$, but not in every case, therefore, we have to check whether $U$ is indeed increased when we maximize $\mathbf{u}_{\mathrm{i}} \mathbf{u}_{\mathrm{i}+1}+\mathbf{u}_{\mathrm{k}} \mathbf{u}_{\mathrm{k}+1}$.

When ${ }^{\mathrm{R}} \mathbf{p}_{i}$ is a point in the relative coordinate system $R$ ', and $\mathbf{v}_{\mathrm{i}}, \ldots, \mathbf{v}_{\mathrm{k}}, \mathbf{v}_{\mathrm{k}+1}$ are the projections of the vectors onto a plane perpendicular to the vector $\mathbf{p}_{\mathrm{i}}-\mathbf{p}_{\mathrm{k}}$, the expression to be maximized is: $\max \left\{\mathbf{v}_{\mathrm{i} \cdot} \mathbf{v}_{\mathrm{i}+1}+\mathbf{v}_{\mathrm{k}} \cdot \mathbf{v}_{\mathrm{k}+1}\right\}$, or in fully developed form:

$$
\max \left\{\left({ }^{\mathrm{R}^{\prime}} \mathbf{p}_{\mathrm{i}}-{ }^{\mathrm{R}} \mathbf{p}_{\mathrm{i}-1}\right)\left({ }^{\mathrm{R}} \mathbf{p}_{\mathrm{i}+1}(\alpha)-{ }^{\mathrm{R}} \mathbf{p}_{\mathrm{i}}\right)+\left({ }^{\mathrm{R}} \mathbf{p}_{\mathrm{k}}-{ }^{\mathrm{R}} \mathbf{p}_{\mathrm{k}-1}(\alpha)\right)\left({ }^{\mathrm{R}} \mathbf{p}_{\mathrm{k}+1}-{ }^{\mathrm{R}} \mathbf{p}_{\mathrm{k}}\right)\right\}
$$

After transforming the resultant curve into the plane, we have to rotate a point $\mathbf{p}_{j+1}$ around the point $\mathbf{p}_{\mathrm{j}}$, and an another point $\mathbf{p}_{\mathrm{k}-\mathrm{l}}$ around $\mathbf{p}_{\mathrm{k}}$ in such a way that the distance between the rotated points $\mathbf{p}_{j+1}$ ' and $\mathbf{p}_{\mathrm{k}-1}{ }^{6}$ should remain equal to the original distance between the points $\mathbf{p}_{j+1}$ and $\mathbf{p}_{k-1}$. The distance can be calculated by $\left\|\mathbf{p}_{i+1}-\mathbf{p}_{\mathrm{k}-1}\right\|=\left\|\mathbf{p}_{\mathrm{i}+1}{ }^{\prime}-\mathbf{p}_{\mathrm{k}-1}{ }^{\prime}\right\|$. Let the angle between the vectors $\mathbf{p}_{\mathrm{i}+1}-\mathbf{p}_{\mathrm{i}}$ and $\mathbf{p}_{\mathrm{i}+1}{ }^{\prime}-\mathbf{p}_{\mathrm{i}}$ be denoted by $\theta$ and, similarly, the angle between the vectors $\mathbf{p}_{\mathrm{k}-1}-\mathbf{p}_{\mathrm{k}}$ and $\mathbf{p}_{\mathrm{k}-1}{ }^{\prime}-$ $\mathbf{p}_{\mathrm{k}}$ denoted by $\beta$. Then, $\mathbf{p}_{\mathrm{i}+1}{ }^{\prime}=f(\theta)$, and $\mathbf{p}_{\mathrm{k}-1}{ }^{\prime}=f(\beta)$. Hence, the expression to be maximized is:

$$
\max \left(\mathbf{u}_{\mathrm{i}-1} \cdot \mathbf{u}_{\mathrm{i}}+\mathbf{u}_{\mathrm{i}} \cdot \mathbf{u}_{\mathrm{i}+1}+\mathbf{u}_{\mathrm{k}-1} \cdot \mathbf{u}_{\mathrm{k}}+\mathbf{u}_{\mathrm{k}} \cdot \mathbf{u}_{\mathrm{k}+1}\right)
$$


It contains two unknown variables $\theta$ and $\beta$ which can be computed analytically.

The computation described above is however rather laborious, therefore the authors applied a numerical procedure to find the values. The requested position of $\mathbf{p}_{\mathrm{k}-1}$ is calculated as one of the two possible intersection points of two circles around $\mathbf{p}_{\mathrm{i}+1}$ ' and $\mathbf{p}_{\mathrm{k}}$. For easier calculation, the circles are transformed into a common system of coordinates. As a solution the smallest value of $\beta$ is selected from the computed values. Since the requested change cannot be properly predicted, an iteration is used to find the maximum value specified by the former expression. The value of $\theta$ is set to a sufficiently small value (in our case: 0.1 ) and the value of the expression is recalculated. Rotation is applied whenever the computed value increases, and the original position is kept otherwise. Then, the value of $\theta$ is decreased to its half unless it has reached a threshold value ( 0.0001 rads).

An important issue related to the profile curve calculation is the estimation of the needed blade length. There are four geometric factors that have significant effect on the requested length of the blade: (a) the layer thickness to be applied, (b) the blade access directions adaptable to the front surface of a layer, (c) the curvedness of the front surface e, and (d) the allowed deviation $\delta$ between the shape curve $\mathbf{q}$ and the profile curve $\mathbf{r}$. Apparently, an unfavorable blade matching direction results in large working length for the blade. Nevertheless, this problem cannot be treated on the level of slicing planning, i.e., objects of detrimental morphological characteristics must be segmented prior to slicing. Considering mechanical aspects such as the risk of loosing the physical stability and stiffness, the length of the blade should be kept down to its possible minimum. Since we intend to cut different curved sectors with the same flexible blade, the set blade length is supposed to allow us to change the tool position. Obviously, cutting of different sectors of the object means change in the instantaneous positions of the reference point $\mathbf{t}$ of the blade. Under these circumstances only an estimate can be given for the initial length of the flexible blade.

\section{ALGORITHM FOR SLICING THE OBJECT INTO LAYERS OF STANDARD THICKNESS}

The layer thickness calculation uses the result of the curve generation together with a heuristic curve matching. We assume that a set $\mathrm{W}$ of layers of discrete (standard) thickness is available such as $W=\left\{w_{1}, \ldots, w_{n}\right\}$. Let's also assume that the decomposition of the object has happened and all of the available segments are well-shaped. The algorithm presented below is targeted to one particular segment and proceeds through the steps as follows: Assume that a base plane $\mathbf{f}$ has been found that can be used as a bottom surface for the very first layer and that it satisfies the requirements for the best slicing position. Furthermore, let the segment bounded by some surface $\mathbf{e}$, and then:

1) Take the boundary curve $\mathbf{s}$ of the segment in the plane $\mathbf{f}$.

2) Find the curvature zones $\Delta \kappa_{j}$, where $0 \leq j \leq \mathrm{N}$, in a band of width $1.5 w_{\max }$ along the boundary curve $s$ in which the normal curvature in the longitudinal direction is moderately variant. It is useful to note that a curvature zone $\Delta \kappa_{j}$ is considered moderately variant if the curvatures $\kappa_{j}$ of two adjacent curves $\mathbf{q}_{\mathbf{j}+1}$ and $\mathbf{q}_{\mathbf{j}}$ at all of their corresponding points (sampled at distances proportional to their length) do not differ by more than a limit value $\eta$. That is, $\eta \geq \Delta \kappa_{j, \max }=\kappa_{j+1, k}-\kappa_{j, k}$ and in practice $\eta \approx 0.1$ is a good choice.

3) Let's indicate the limits of the zones by $k_{j}$ For the shape curve $q_{j}$ at $k_{j}$ generate an approximating profile curve $\mathbf{r}_{\mathbf{j}}$.

4) Place one arbitrary point $\mathbf{b}_{r, j}$ of the profile curve $\mathbf{r}_{j}$ over the basis point $\mathbf{b}_{q, j}$ of the shape 
curve $\mathbf{q}_{\mathbf{j}}$. Let's call this point the common point $\mathbf{b}$ from now on.

5) Rotate the profile curve $\mathbf{r}_{\mathbf{j}}$ around the common point $\mathbf{b}$ until minimum one more common (intersection) point is found and the deviation $\delta$ between the corresponding points of curves $\mathbf{q}_{\mathbf{j}}$ and $\mathbf{r}_{\mathbf{j}}$ is less than a $\delta_{0}$. Call this point the point $\mathbf{c}$ afterwards.

6) If the matching described in the Step 5 failed, go to the Step 3.

7) Find the distances $d_{j}$ between the points $\mathbf{c}$ on curves $\mathbf{q}_{\mathbf{j}}$ and the plane $\mathbf{f}$ by an orthonormal projection.

8) Compare the distances $d_{j}$ to the maximum layer thickness $w_{\max }$. If all $d_{j}$ are bigger than $w_{\text {max }}$, cut the segment by a plane $\mathbf{g}$, parallel to $\mathbf{f}$, at a distance $w_{\max }$. Otherwise, use that particular layer thickness $w_{\text {appl }}$ for which $\varepsilon=\left(d_{j}-\mathrm{w}_{a p p l}\right)$ is minimum and $\varepsilon>0$.

9) Calculate the intersection points $\mathbf{c}^{\prime}$ for all curves $\mathbf{q}_{\mathbf{j}}$. Check the extent of the remaining part of the segment. If it is bigger than $w_{\min }$ consider the plane $\mathbf{g}$ as a new plane $\mathbf{f}$ and go to the Step 1. Otherwise continue with the Step 10.

10) Find the minimum of the distances $d_{j}$ and find the layer thickness for which $d_{j, \min } \geq w$. If $d_{j, \min }<\mathrm{w}_{\min }$ then stop. Otherwise cut the segment by a plane $\mathbf{g}$, parallel to $\mathbf{f}$, at a distance $w$.

11) Calculate the intersection points $\mathbf{c}^{\prime}$ for all curves $\mathbf{q}_{\mathrm{j}}$. Check the extent of the remaining part of the segment. If it is bigger than $w_{\min }$ consider the plane $\mathbf{g}$ as a new plane $\mathbf{f}$ and go to the Step 1. Otherwise indicate insufficiency and stop.

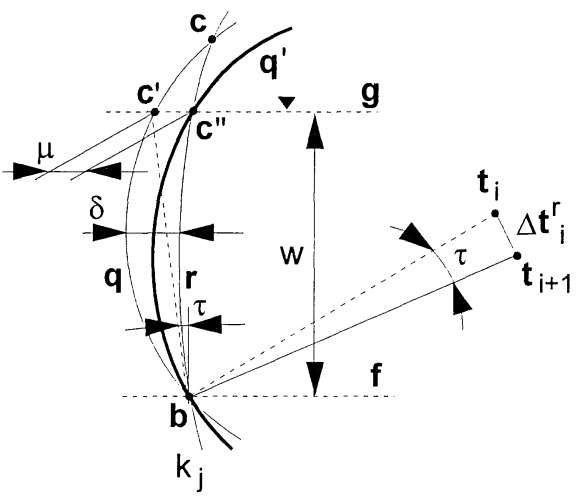

Figure 4. The principle of error compensation for standard layers

At the end of this process, the segment is cut into layers whose front surfaces approximate the pertaining nominal surface of the CAD model with a deviation less than $\delta$. However, when layers of standard thickness are used instead of the computed ones, reposition of the cutting plane from the point $\mathbf{c}$ to the point $\mathbf{c}$ ' is needed. However, by moving the cutting plane an overrunning step is formed since calculation for the next layer starts out of the nominal boundary curves again. When a standard layer thickness is used as a lower bound, the actual intersection points c' on the curve $\mathbf{q}$ and $\mathbf{c}$ " on the curve $\mathbf{r}$ will substitute the nominal intersection point $\mathbf{c}$ (Figure 4). For instance, when $d_{\min } \geq w_{\max }$ the extent of the upper surface $\mathbf{g}$ will be larger or smaller than the one received when the points $\mathbf{c}$ ' are exactly at a distance $w_{\text {max }}$. We call the distance between the actual points $\mathbf{c}$ ' and the theoretical places of the points $\mathbf{c}$ at the same height $w$ from the bottom plane $\mathbf{f}$ (i.e., the point $\mathbf{c}$ ") a layer boundary 
repositioning error $\mu$. It has to be compensated for in order to receive at least quasi- $C^{l}$ continuity at the interface of the neighboring layers. It means that the point c" have to be moved to the points $\mathbf{c}^{\prime}$ with the calculated $\mu_{j}$ in the direction of $\overline{c^{\prime} c^{\prime \prime}}$. Note that rotation of the curves $\mathbf{r}_{\mathbf{j}}$ generally results in under-curved or over-curved approximation. It can be proved however that the possible maximum value of $\mu_{j}$ is $\mu_{\max } \leq \delta$ and it can be taken into consideration at the initial selection of the maximum value for $\delta$.

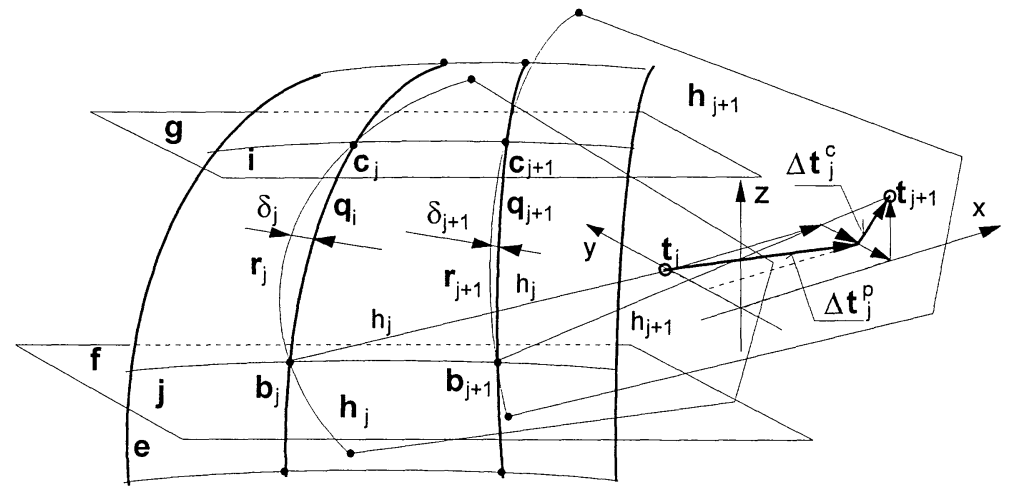

Figure 5. Calculation of the actual position of the reference point of the flexible blade in a particular situation

\section{ALGORITHM FOR TOOL POSITION AND TOOL PATH CALCULATION}

A given functional, geometric and positional relationship between a layer to be fabricated and the flexible blade/tool at a given moment of time is called situation. For computational reasons, the continuous process of layer fabrication should be broken into a given sequence of discrete situations. The requested number of situations to compute is jointly influenced by the shape of the object and the preciseness to be achieved. For constraining the requested number of computed situations, a threshold value is applied in the change in the curvature. There are three reasons of translocations (change in the tool position) in between two subsequent situations: (a) the profile curve $\mathbf{r}_{j}$ of the flexible blade has to follow the variation of the shape curve $\mathbf{q}_{\mathrm{j}}$ to keep the allowed deviation $\delta$, (b) the tool has to follow the boundary curve $\mathbf{s}$ of the front surface $\mathbf{e}$ of a layer, and (c) the tool has to be rotated around the point $\mathbf{b}_{j}$ to compensate for the layer boundary repositioning error $\sigma$ (Figure 5 ).

The tool position calculation decomposes into four tasks, namely, finding the values of (a) the longitudinal translocation $\Delta \mathbf{t}^{\mathrm{c}}{ }_{\mathrm{j}}$ due to the resetting of the blade, (b) the longitudinal translocation $\Delta \mathbf{t}_{\mathrm{j}}^{\mathrm{r}}$ due to the tilting of the blade to compensate for the repositioning error, and (c) the circumferential translocation $\Delta \mathbf{t}^{\mathrm{p}}{ }_{\mathrm{j}}$ due to the tool alignment, and (d) generating the resultant translocation $\Delta \mathbf{t}_{j}$ of the reference point $\mathbf{t}$ of the tool. While the longitudinal translocation at a given zone-end $k_{j}$.can be calculated from the change in the relative positions of the curves $\mathbf{q}_{j}$ and $\mathbf{r}_{j}$, for calculation of the circumferential translocation we need to know 
the boundary curve s of the layer, that is obtained after a previous slicing. The algorithm for calculation of the change in the tool positions due to resetting works as follows. Knowing the tool geometry and the reference point a of the curve $\mathbf{r}_{j}$ the absolute position of the reference point $\mathbf{t}_{j}$ of the tool is $\mathbf{t}_{j}=\mathbf{a}+\mathbf{h}$. However, the actual position of curve $\mathbf{r}_{j}$ is determined by its fitting to the curve $\mathbf{q}_{j}$. Considering that the location of the reference point $\mathbf{v}_{j}$ of the curve $\mathbf{q}_{j}$ is known (since the object is supposed to be positioned in a system of coordinates), the vector $\mathbf{t}_{\mathbf{j}}$ in a $\mathbf{j}$-th situation can be expressed as $\mathbf{t}_{j}^{\mathrm{c}}=\mathbf{v}+(\mathbf{b}-\mathbf{v})_{j}+(\mathbf{a}-\mathbf{b})_{j}+\mathbf{h}$. Furthermore, for the $(\mathrm{j}+1)$-th situation it is $\mathbf{t}_{\mathbf{j}+1}^{\mathrm{c}}=\mathbf{v}+(\mathbf{b}-\mathbf{v})_{\mathbf{j}+1}+(\mathbf{a}-\mathbf{b})_{\mathbf{j}+1}+\mathbf{h}$. The difference is $\Delta \mathbf{t}_{j}^{\mathrm{c}}=\mathbf{t}^{\mathrm{c}}{ }_{\mathrm{j}+1}-\mathbf{t}_{\mathbf{j}}^{\mathrm{c}}$, or in an extendeded form:

$$
\Delta \mathbf{t}_{j}^{\mathrm{c}}=\left((\mathbf{b}-\mathbf{v})_{\mathrm{j}+1}-(\mathbf{b}-\mathbf{v})_{j}\right)+\left((\mathbf{a}-\mathbf{b})_{j+1}-(\mathbf{a}-\mathbf{b})_{\mathrm{j}}\right)
$$

In principle, there are several ways of compensating for the repositioning errors $\mu_{j}$. We have adapted the one which is the least computation-intensive. Actually the curve generation and matching procedure is not repeated, but the needed compensation is achieved by modification of the tool position in harmony with the errors $\mu_{j}$. It can be also seen in Figure 4 . The angle of the needed rotation, $\tau$, of the tool around the common point $\mathbf{b}$ can be calculated by: $\tau=\operatorname{arctg}(\mu / w)$, where $\mu$ is the distance of $\overline{c c^{\prime \prime}}$. The rotation $\tau$ results in a displacement $\Delta t^{r}$ for the tool's reference point $\mathbf{t}_{j}$. To calculate the longitudinal translocation $\Delta t_{j}^{r}$, let's denote the position of the reference point $\mathbf{t}_{j}$ before rotation of the matching curve $\mathbf{r}_{j}$ by $\mathbf{t}_{j}{ }_{j}$ and the position after rotation by $\mathbf{t}_{j+1}^{\mathrm{r}}$ at a given $k_{j}$. The actual value of $\Delta \mathrm{t}^{\mathrm{r}}{ }_{\mathrm{i}}$ can be calculated by $\Delta \mathbf{t}^{r}=\mathbf{t}^{r}{ }_{j+1}-\mathbf{t}_{j}{ }_{j}$. Knowing the angle of rotation $\tau$, and $\mathbf{t}_{j}^{r}$, we can calculate the new position by the expression $\mathbf{t}_{j+1}^{r}=\mathbf{v}+(\mathbf{b}-\mathbf{v})_{j}+\mathbf{R} \times\left((\mathbf{a}-\mathbf{b})_{j}+\mathbf{h}\right)$, where $\mathbf{R}$ is a $2 \times 2$ rotation matrix of $\tau$. Finally, the longitudinal translocation $\Delta \mathbf{t}^{\mathrm{r}}$ is given by

$$
\Delta \mathbf{t}_{\mathrm{j}}^{\mathrm{r}}=\left(\mathbf{R} \times(\mathbf{a}-\mathbf{b})_{\mathrm{j}}+\mathbf{h}\right)-\left((\mathbf{a}-\mathbf{b})_{\mathrm{j}}+\mathbf{h}\right) \text {. }
$$

Since $\Delta \mathbf{t}^{\mathrm{c}}{ }_{\mathrm{j}}$ and $\Delta \mathbf{t}_{\mathrm{j}}^{\mathrm{r}}$ are coplanar vectors, i.e., they are in the plane $\mathbf{h}$ of the blade, their vectorial sum is also a planar vector.

The circumferential translocation $\Delta \mathbf{t}^{\mathrm{p}}$ is determined by from two subsequent positions of the principal plane $\mathbf{h}$ of the blade/tool along a boundary curve $\mathbf{s}$, as required by the local circumferential curvatures. The actual value of $\Delta \mathrm{t}^{\mathrm{p}}{ }_{\mathrm{i}}$ can be calculated by the formula: $\Delta \mathbf{t}^{\mathrm{p}}{ }_{\mathbf{j}}=\mathbf{t}^{\mathrm{p}}{ }_{\mathrm{j}+1}-\mathbf{t}^{\mathrm{p}}{ }_{\mathbf{j}}$ The place $\mathbf{t}^{\mathrm{p}}$ of the reference point in the $(\mathrm{j}+1)$-th situation is: $\mathbf{t}^{\mathrm{p}}{ }_{\mathbf{j}+1}=\mathbf{b}_{\mathbf{j}+1}+\mathbf{R}\left(\beta_{\mathbf{j}+1}\right) \times\left(\mathbf{R}\left(\alpha_{\mathbf{j}+1}\right) \times\left(\mathbf{t}_{\mathbf{j}+1}-\mathbf{b}_{\mathbf{j}+1}\right)\right)$. In the $\mathrm{j}$-th situation $\mathbf{t}^{\mathrm{p}}{ }_{\mathbf{j}}$ can be expressed by $\mathbf{t}^{\mathrm{p}}{ }_{\mathrm{j}}=\mathbf{b}_{\mathbf{j}}+\mathbf{R}\left(\beta_{\mathbf{j}}\right) \times\left(\mathbf{R}\left(\alpha_{\mathbf{j}}\right) \times\left(\mathbf{t}_{\mathbf{j}}-\mathbf{b}_{\mathbf{j}}\right)\right)$. In the plane $\mathbf{f}$ the circumferential translocations is:

$$
\Delta \mathbf{t}_{\mathrm{j}}^{\mathrm{p}_{\mathrm{j}}}=\left(\mathbf{b}_{\mathrm{j}+1}-\mathbf{b}_{\mathrm{j}}\right)+\left(\mathbf{R}\left(\beta_{\mathrm{j}+1}\right) \times\left(\mathbf{R}\left(\alpha_{\mathrm{j}+1}\right) \times\left(\mathbf{t}_{\mathrm{j}+1}-\mathbf{b}_{\mathbf{j}+1}\right)\right)-\right.
$$




$$
\left.-\mathbf{R}\left(\beta_{\mathbf{j}}\right) \times\left(\mathbf{R}\left(\alpha_{\mathbf{j}}\right) \times\left(\mathbf{t}_{\mathbf{j}}-\mathbf{b}_{\mathbf{j}}\right)\right)\right) .
$$

The resultant translocation $\Delta \mathbf{t}_{\mathbf{j}}$ can be calculated as a vectorial sum:

$$
\Delta \mathbf{t}_{j}=\Delta \mathbf{t}^{\mathrm{c}}{ }_{\mathrm{j}}+\Delta \mathbf{t}_{\mathrm{j}}^{\mathrm{r}}+\Delta \mathbf{t}^{\mathrm{p}}{ }_{\mathrm{j}}
$$

The requested movement (translations and rotations) of the tool must be described in the global system of coordinates $\Gamma$ of the six-axis sculpturing robot, used for thick-layered freeform manufacturing. First, to get the absolute positions of the contacts points of the flexible blade, the calculated relative displacements have to be transformed into the system of coordinates $\Gamma$ with reference to its origin. The flexible cutter is controlled electronically to follow the computed tool path, while the shape of the flexible blade is modified electromechanically.

\section{VISUALIZATION OF THE TLOM SUBPROCESSES}

The programs for profile curve calculation, object slicing, tool position and tool path calculation were coded in standard $\mathrm{C}++$ language. For visual presentation of the computed results the Open Inventor graphical programming environment was used on a Silicon Graphics workstation.

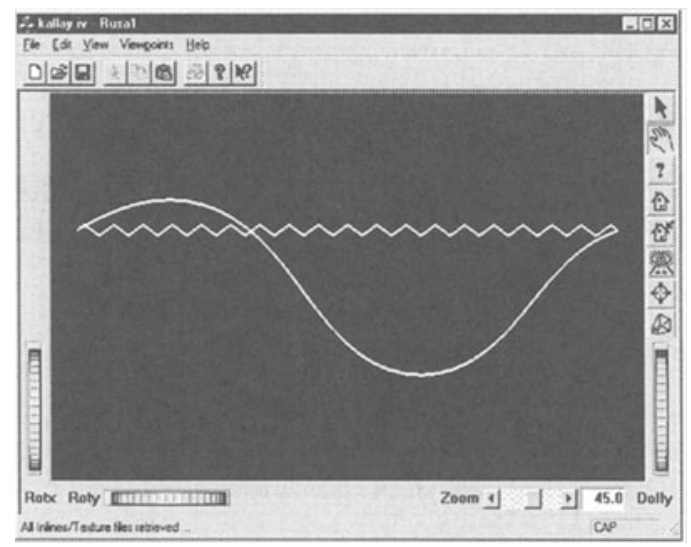

Figure 6. Visualization of the profile curve computation

The shape of the flexible blade is generated/calculated with the assumption of minimum strain energy and specifying the boundary conditions in terms of positions of the supports and tangency at the supports. The profile curve generation algorithm works in two phases. First, it operates in space to find locations of the points approaching to the smoothest curve. Then, based on the obtained approximate curve, it continues with looking for the optimum (least energy) planar curve in the middle plane of the flexible blade tool. The displayed results of shape computation can be seen in Figure 6. The computed visual model of the flexible blade is used practically in all steps of the virtual simulation of the layer cutting process. 


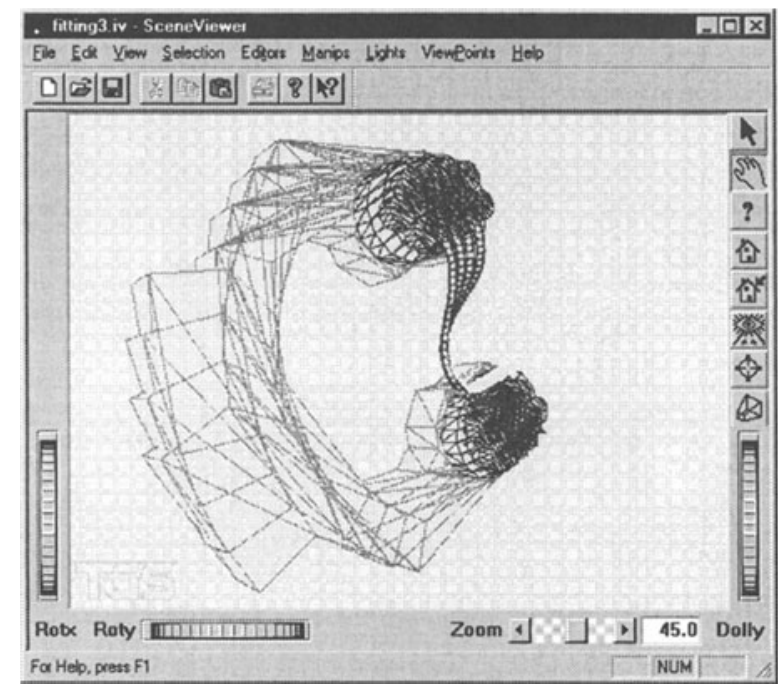

Figure 7. Matching the tool profile curve to a shape curve

The parameters that influence the resultant shape of the profile curve are (a) the length of the blade, (b) the distance between the support points of the blade, and (c) the tangent vectors at the start and end point of the blade. In order to decrease the in-process calculation time, a database of profile curves has been created by specifying appropriate values to the main parameters. To support selection, ten equidistant points have been sampled on each curve and the local curvature calculated. Based on the curvature information, the curve which promises the best fitting to a given shape curve can be easily selected and used for matching.

Different sectors of the profile curves are used to find the best fitting (Figure 7). The developed software tries to use a particular curve for approximation of as many shape curves along the periphery of a potential layer as possible at all. This way the number of tool changes and resetting can be decreased. Figure 8 shows how the front surface of a layer is cut out by a sequence of profile curves.

In the Section 4, the instantaneous tool positions were described relative to the nominal surfaces of the layer being manufactured. A set of discrete tool reference points was calculated. These points, together with one or more blade-layer contact points specify the place and position of the tool frame. Since in our case the flexible blade tool is held and moved by the six-axis sculpturing robot, the tool positions recomputed in the global frame $\Gamma$ of the sculpting robot. It can be achieved by applying concatenated transformation matrices to all of the robot elements and the flexible blade tool ${ }^{14}$.

Further research is needed to optimize the process parameters and the tool path and, hence, the obtained quality. The on-going work focuses on the following issues of tool path generation: (a) consideration positional tolerances in calculation of feature points of the tool path, (b) generating smooth tool path by curve interpolation of the calculated tool positions, (c) optimization of the resultant tool path in terms of the minimal requested motion control, and (d) simulation of the front surface manufacturing process for each layer. As well, the visual simulation of the TLOM process is intended to present the segment and layer assembling subprocess (virtual recomposition) in order to reveal possible problems with positioning, stacking and fixing. However, the study of the methods for controlling the human-implemented stacking have just been recently started. 


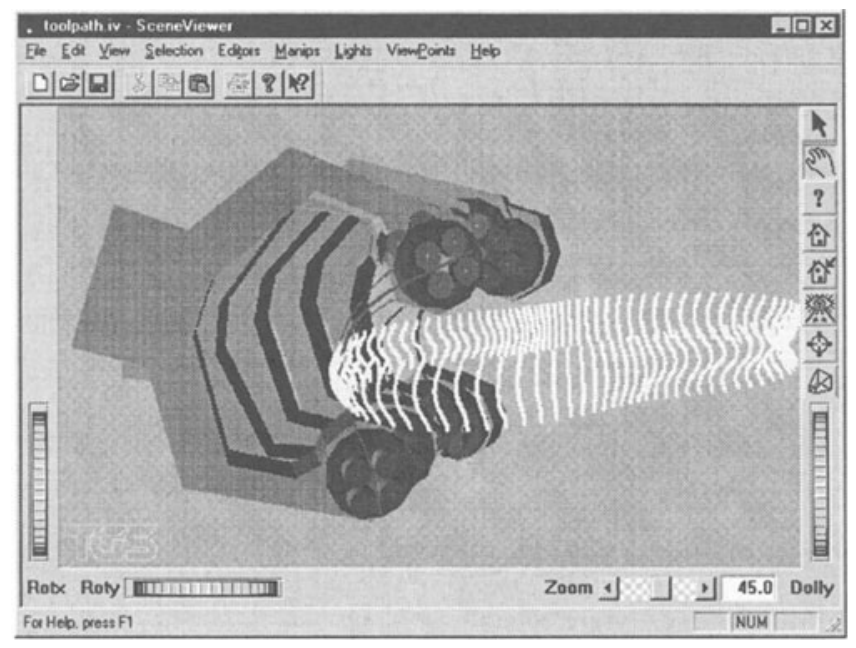

Figure 8. The sequence of tool positions for layer manufacturing

\section{CONCLUSIONS}

The objective of our research and development has been to come up with an effective technology for fabrications of large sized, free-form physical models of various soft materials based on higher order shape approximation. This technology proved to be feasible. To find the profile curve of the blade we successfully applied an extended version of the discrete point based minimum energy curve calculation method, first published by M. Kallay. In order not to exceed the allowed deviation value, a curve matching technique that manipulate on the best fitting part of the generated profile curve has been applied. Our slicing algorithm considers standard layer thickness and takes the repositioning errors occurring on the periphery of the layers into consideration as well. The resultant continuity in the internal domains of the front surfaces of the fabricated object is $C^{l}$ in longitudinal direction, and quasi- $C^{l}$ in circumferential direction. To get the optimal cutting results, a manufacturing equipment of high kinematical degrees of freedom has been used. Further work will focus on the optimization of the process parameters, further parallelization of the shape calculations and tool path calculations.

\section{REFERENCES}

1 Cox, M. G.,"Data Approximation by Splines in One and Two Independent Variables", in NPL Report DITC 7/86, National Physics Laboratory, Teddington. (1986).

2 de Jager P. J., Broek, J. J., Vergeest, J. S. M., "A Comparison Between Zero and First Order Approximation Algorithms for Layered Manufacturing", Assembly Automation, Vol. 17, No. 3, (1997), pp. 233-238.

3 de Jager, P. J., "Development of a New Slicing Methodology to Improve Layered Manufacturing", Ph.D. Thesis, Delft University of Technology, Delft, (1997), pp. 1-118.

4 Frisch-Fay, R., "Flexible Bars", Butterworth \& Co., London, (1962).

5 Guduri, S., Crawford, R. H., Beaman, J. J., "A Method to Generate Exact Contour Files for Solid Freeform Fabrication", in Proceedings of Solid Freeform Fabrication Symposium 1992, ed. by Marcus, H. L. et al., University of Texas, Austin, August 1992, pp. 95-101.

6 Hope, R. L., Riek, A. T., Roth, R. N., "Layer Building with Sloped Edges for Rapid Prototyping of 
Large Objects", in Proceedings of the 5th European Conference on Rapid Prototyping and Manufacturing, ed. by Dickens P., Helsinki, (1996), pp. 47-58.

7 Hope, R. L., Jacobs, P. A., Roth, R. N., "Rapid Prototyping with Sloping Surfaces"; Rapid Prototyping Journal, Vol. 3, No. 1, (1997), pp. 12-19.

8 Horn, B. K. P., "The Curve of Least Energy", ACM Transactions of Mathematics and Software, Vol. 9, No. 4, (1983), pp. 441-460.

9 Horváth, I., Vergeest, J. S. M., Broek, J. J., de Smit, A., "Tool Profile and Tool Path Calculation for Free-form Thick-layered Fabrication", Computer Aided Design, Vol. X, No. Y, (1998), pp. 26, (Status: accepted for publication).

10 Horváth, I., Vergeest, J. S. M., Juhász, I.: "Finding the Shape of a Flexible Blade for Free-form Layered Manufacturing of Plastic Foam Objects", in Proceedings of 1998 ASME Design Engineering Technical Conferences DETC'98, September 13-16, 1998 - Atlanta, Georgia, CD-ROM: DETC98/DFM-5752, ASME, New York.

11 Jamieson, R., Hacker, H., "Direct Slicing of CAD Models for Rapid Prototyping", Rapid Prototyping Journal, Vol. 1, No. 2, (1995), pp. 4-12.

12 Jerome, J. W., "Smooth Interpolating Curves of Prescribed Length and Minimum Curvature", Proceedings of the American Mathematics Society, Vol. 51, No. 1, (1975), pp. 62-66.

13 Kallay, M., "Method to Approximate the Space Curve of Least Energy and Prescribed Length", Computer Aided Design, Vol. 2, No. 3, (1987), pp. 73-76.

14 Kuczogi, Gy., Rusák, Z., Kovács, Zs., "Report on Visualization of Free-Form Thick Layered Object Fabrication", Internal Communication, Delft University of Technology, ICA Project, (1998), pp. 18.

15 Kulkarni, P., Dutta, D., "Adaptive Slicing of Parametrizable Algebraic Surfaces for Layered Manufacturing", in Proceedings of the ASME Design Engineering Technical Conferences, Vol. 1, ed. by Azram, S. et al., ASME, New York, (1995), pp. 211-217.

16 Kulkarni, P., Dutta, D., "An Accurate Slicing Procedure for Layered Manufacturing", Computer Aided Design, Vol. 28, No. 9, (1996), pp. 683-697.

17 Lee, E. H., Forsythe, G. E., "Variational Study of Nonlineaer Curves", SIAM Review, Vol. 15, No. 1, (1973), pp. 120-133.

18 Sabourin, E., Houser, S. A., Bohn, J. H., "Adaptive Slicing Using Stepwise Uniform Refinement", Rapid Prototyping Journal, Vol. 2, No. 4, (1996), pp. 20-26,

19..Suh, Y. S., Wozny, M. J., "Adaptive Slicing of Solid Freeform Fabrication Processes", in Proceedings of the Solid Freeform Fabrication Symposium '94, ed. by Marcus, H. L. et al., The University of Texas at Austin, Austin, (1994), pp. 404-411.

20 Vergeest, J. S. M., "Surface Fitting for Interactive Shape Design", Computers in Industry, Vol. X, No. 13, (1989), pp. 1-13.

21 Vuyyuru, P., Kirschman, C. F., Fadel, G. Bagchi, A., Jara-Almonte, C. C., "A NURBS-Based Approach For Rapid Product Realization," Proceedings of the Fifth International Conference on Rapid Prototyping, ed by Chartoff, R. P. et al, The University of Dayton, June 1994, pp. 229-239. 